\title{
Extended $V$-systems and trigonometric solutions to the WDVV equations
}

\author{
Cite as: J. Math. Phys. 62, 022301 (2021); doi: 10.1063/5.0024108 \\ Submitted: 4 August 2020 - Accepted: 29 November 2020 • \\ Published Online: 1 February 2021
}

\section{Richard Stedman ${ }^{\text {a) }}$ and lan A. B. Strachan}

\section{AFFILIATIONS}

School of Mathematics and Statistics, University of Glasgow, Glasgow G12 8QQ, United Kingdom

a) richard.stedman@gmail.com

${ }^{b)}$ Author to whom correspondence should be addressed: ian.strachan@glasgow.ac.uk

\begin{abstract}
Rational solutions of the Witten-Dijkgraaf-Verlinde-Verlinde (or WDVV) equations of associativity are given in terms of configurations of vectors, which satisfy certain algebraic conditions known as $\bigvee$-conditions [A. P. Veselov, Phys. Lett. A 261, 297-302 (1999)]. The simplest examples of such configurations are the root systems of finite Coxeter groups. In this paper, conditions are derived that ensure that an extended configuration - a configuration in a space one-dimension higher-satisfies these $\bigvee$-conditions. Such a construction utilizes the notion of a small orbit, as defined in Serganova [Commun. Algebra, 24, 4281-4299 (1996)]. Symmetries of such resulting solutions to the WDVV equations are studied, in particular, Legendre transformations. It is shown that these Legendre transformations map extended-rational solutions to trigonometric solutions, and for certain values of the free data, one obtains a transformation from extended $\vee$-systems to the trigonometric almost-dual solutions corresponding to the classical extended affine Weyl groups.
\end{abstract}

Published under license by AIP Publishing. https://doi.org/10.1063/5.0024108

\section{INTRODUCTION}

\section{A. Background}

Solutions of the form

$$
F=\frac{1}{4} \sum_{\alpha \in \mathcal{U}} h_{\alpha} \alpha(z)^{2} \log \alpha(z)
$$

of the Witten-Dijkgraaf-Verlinde-Verlinde (or WDVV) equations have appeared in many different settings, for example,

- $\quad$ in Seiberg-Witten theory; ${ }^{1}$

- in the theory of almost-dual Frobenius manifolds; ${ }^{2}$

- $\quad$ on the space of stability conditions; ${ }^{3}$

- $\quad N=4$ superconformal many-particle quantum mechanics. ${ }^{4,5}$

Here, $\mathcal{U}$ is a finite collection of covectors, and $h_{\alpha}$ are numbers-known as multiplicities-associated with each covector. In the first examples of this type, the sets $\mathcal{U}$ were the collections $\mathcal{R}_{W}$ of root vectors of a finite Coxeter groups $W$, and in order to understand and generalize the construction of these solutions, Veselov introduced the notion of a $\bigvee$-system. ${ }^{6}$ Roughly (and a precise definition will follow), in order for (1) to satisfy the WDVV equations (which are a set of over-determined partial differential equations), the covectors in $\mathcal{U}$ have to satisfy certain algebraic conditions, and if these are satisfied, the configuration is said to be a $\bigvee$-system. If the multiplicities are positive, they may be absorbed into the covectors, but if some are negative, such a procedure breaks the geometry or rather moves it into the complex, so in this paper, we keep the multiplicities separate. Thus, for us, a $\bigvee$-system consists of pairs $\left(\alpha, h_{\alpha}\right)$, consisting of a covector and its associated multiplicity, which satisfy the $\bigvee$-conditions (which are given in Definition 3 ).

The origin of this paper was to understand the geometry of the configurations, which define a certain solution of the WDVV equation of almost-dual type. Starting with a superpotential (see Sec. V) 


$$
\lambda(z)=\left.\prod_{i=0}^{N}\left(z-z^{i}\right)^{k_{i}}\right|_{\sum_{i=0}^{N} k_{i} z^{i}=0},
$$

one obtains the solution

$$
F=\left.\frac{1}{4} \sum_{i \neq j} k_{i} k_{j}\left(z^{i}-z^{j}\right)^{2} \log \left(z^{i}-z^{j}\right)\right|_{\sum_{i=0}^{N} k_{i} z^{i}=0}
$$

with metric $g=\left.\sum_{i=0}^{N} k_{i} d\left(z^{i}\right)^{2}\right|_{\sum k_{i} z^{i}=0}$, and from the superpotential

$$
\lambda(z)=z^{s} \prod_{i=1}^{N}\left(z^{2}-\left(z^{i}\right)^{2}\right)^{k_{i}}
$$

one obtains the solution

$$
F=\sum_{i=1}^{N} k_{i}\left(s+2 k_{i}\right)\left(z^{i}\right)^{2} \log z^{i}+\frac{1}{2} \sum_{i \neq j} k_{i} k_{j}\left(z^{i} \pm z^{j}\right)^{2} \log \left(z^{i} \pm z^{j}\right),
$$

with metric $g=\sum_{i=1}^{N} k_{i} d\left(z^{i}\right)^{2}$ (note that this metric is independent of the value of $s$ ). In the cases when $k_{i}=1$ for all $i$, these are the well-known $A_{N}$ and (for $\left.s=0\right) B_{N}$ solutions, with a configuration being the root vectors of these Coxeter groups. Introducing multiplicities either in the zeros or poles of the superpotential-depending on the sign of $k_{i}$-destroys this interpretation and also introduces a split signature metric. From the analysis of the case when

$$
\mathbf{k}^{\text {ext }}=\{k, \underbrace{1, \ldots, 1}_{m}, \underbrace{-1, \ldots,-1}_{n}\},
$$

it was found that the configuration could be interpreted as an extension into a perpendicular direction of the lower dimensional configuration defined by

$$
\mathbf{k}=\{\underbrace{1, \ldots, 1}_{m}, \underbrace{-1, \ldots,-1}_{n}\} \text {. }
$$

The origin of this configuration - which defines a generalized root system-from a rational superpotential aids in the interpretation of its symmetries: the superpotential is invariant under the interchange of zeros and poles. Isotropic roots can be interpreted as the interchange of zeros and poles.

\section{B. Outline}

The purpose of this paper is threefold. First, we construct extended $\bigvee$-systems. Starting with a $\bigvee$-system, we extend the configuration into a one-dimension higher space by adding a one-dimensional orthogonal direction and adding certain special covectors to the original configuration. We then derive the (extra)-conditions required for this extended configuration to be a $\bigvee$-system. This construction utilizes the idea of a small orbit, as introduced by Serganova. Thus, extended $\bigvee$-systems are $\bigvee$-systems, but in one dimension higher than the original system.

Second, we perform a Legendre transformation on an extended $\bigvee$-system. Such Legendre transformations are symmetries of the WDVV equations and hence map solutions to solutions. ${ }^{8}$ To perform such a transformation requires the choice of a direction, and for extended $\checkmark$, there is a natural choice of direction, namely, the newly introduced orthogonal direction perpendicular to the original space. Using this direction for the Legendre transformation results in a transformation that maps rational solutions, i.e., those of form (1), to trigonometric systems, that is, to the solution of the form

$$
F=\text { cubic }+\sum_{\alpha \in \mathcal{U}} h_{\alpha} L i_{3}\left(e^{\alpha(z)}\right)
$$

where $\mathrm{Li}_{3}(z)$ is the tri-logarithm function. A separate theory of trigonometric $\bigvee$-systems has been developed by Feigin ${ }^{9}$ (and for the most results, see Ref. 10).

Finally, we make the connection between extended $\bigvee$-systems and the almost-dual Frobenius manifolds for the extended affine Weyl group orbit spaces, as constructed and studied in Refs. 11 and 12. In particular, the following is proved:

Theorem 1. Let $W$ be a finite irreducible classical Coxeter group of rank $l$, and let $\widetilde{W}$ be the extended affine Weyl group of $W$ with an arbitrary marked node. Then, up to a Legendre transformation, the almost-dual prepotentials of the classical extended affine Weyl group orbit spaces $\mathbb{C}^{l+1} / \widetilde{W}$ are, for specific values of the free data, the extended $\bigvee$-systems of the $\bigvee$-system $R_{W}$.

A more precise version of the theorem-with the full data specified-will be given in Sec. V. 


\section{C. $\vee$-systems and small orbits}

We start by defining a $\bigvee$-system and the $\bigvee$-conditions. Let $V$ be a real vector space and $\mathcal{U} \subset V^{*}$ be a finite set of covectors that span the dual space $V^{*}$. With this, one defines a metric by the formula

$$
\sum_{\alpha \in \mathcal{U}} h_{\alpha} \alpha(x) \alpha(y)=h_{\mathcal{U}}(x, y) .
$$

Here, $h_{\alpha} \in \mathbb{R}$ is the multiplicity of the covector $\alpha$, which may be negative or a non-integer. It is therefore an additional assumption that the metric $(x, y)$ defined in this way is non-degenerate: it certainly can be non-positive definite (see Example 4). For notational convenience, we will sometimes refer to the metric as $g$ rather than $($,$) . The constant h_{\mathcal{U}}$ is technically redundant-it could be absorbed into the multiplicities; however, it is useful to keep this freedom.

As each $\alpha \in \mathcal{U}$ has a corresponding multiplicity $h_{\alpha}$, one could refine $\mathcal{U}$ to be the collection of pairs $\left(\alpha, h_{\alpha}\right)$.

Example 2. With the standard representation of the roots and metric for the Coxeter group $A_{n}$,

$$
\begin{aligned}
& \mathcal{U}=\left\{e_{i}-e_{j}, 0 \leq i, j \leq n, i \neq j\right\}, \\
& g=\left.\sum_{i=0}^{n}\left(d z^{i}\right)^{2}\right|_{\sum z^{i}=0},
\end{aligned}
$$

one has

$$
\sum_{\alpha \in \mathcal{U}} \alpha(x) \alpha(y)=2(n+1)(x, y),
$$

so one takes the normalization $h_{\alpha}=1$ (the roots form a single orbit), which forces $h_{\mathcal{U}}$ to be twice the (dual) Coxeter number.

Again following Ref. 6, this metric defines the isomorphism $\varphi_{\mathcal{U}}: V \rightarrow V^{*}$, and we denote $\varphi_{\mathcal{U}}^{-1}(\alpha)=\alpha^{\vee}$.

Definition 3. The system $\mathcal{U}$ is a $\bigvee$-system if the following relations are satisfied:

$$
\sum_{\beta \in \Pi \cap \mathcal{U}} h_{\beta} \beta\left(\alpha^{\vee}\right) \beta^{\vee}=\lambda \alpha^{\vee}
$$

for each $\alpha \in \mathcal{U}$ and each two-plane $\Pi \subset V^{*}$ containing $\alpha$. The constant $\lambda$ will, in general, depend on both $\Pi$ and $\alpha$.

In the following example, the multiplicities are both positive and negative, and this drastically affects the geometry-the natural metric is no longer positive definite. The example also shows how one can interpret the $\bigvee$-system: one can think of it as the extension of a onedimensional $\bigvee$-system associated with the Coxeter group $A_{1}$ into two dimensions.

Example 4. Consider the following solution to the WDVV equations, ${ }^{13}$

$$
F^{\star}=\frac{1}{4}\left(x^{2} \log x+y^{2} \log y-(x-y)^{2} \log (x-y)\right)
$$

with $g=2 d x d y$. This solution is almost dual to the Frobenius manifold defined by the prepotential

$$
F=\frac{1}{2} t_{1}^{2} t_{2}+t_{2}^{2} \log t_{2} .
$$

The configuration of vectors $\{ \pm(1,0), \pm(0,1), \pm(1,-1)\}$ seems somewhat asymmetric.

However, this does not take into account the split signature of the metric. If one rotates the diagram and superimposes the light-cone, this illuminates the geometry of the configuration.

The vectors $\{ \pm(1,0), \pm(0,1)\}$ are null, and the vectors $\pm(1,-1)$ are space-like (Figs. 1 and 2$)$.

This example also motivates the main construction. We start with a configuration $\mathcal{U}=\{ \pm(1,-1)\}$ spanning a space $V$ (i.e., the configuration is the root system for the Coxeter group $\left.A_{1}\right)$ and extend into a perpendicular direction defined by the normal vector $n=\frac{1}{2}(1,1)$. With this, one obtains an extended space $V^{\text {ext }}$, and an extended space of configurations $\mathcal{U}^{\text {ext }}$ may be constructed by extending certain vectors into the perpendicular direction, so

$$
\begin{aligned}
& (0,1)=\frac{1}{2}(-1,1)+n, \\
& (1,0)=\frac{1}{2}(1,-1)+n .
\end{aligned}
$$




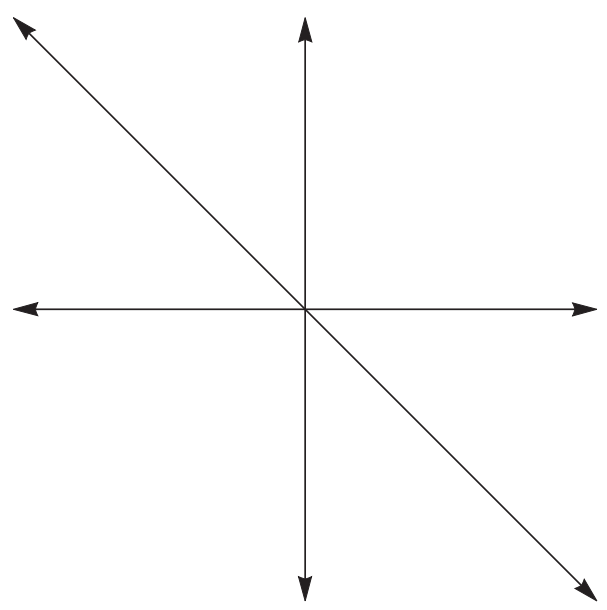

FIG. 1. The geometry of the $\bigvee$-configuration.

What makes the vectors $\pm \frac{1}{2}(1,-1)$ special is the following important property: their difference lies in $\mathcal{U}$. This is known as the small orbit property, and its existence will be crucial to what follows.

Thus, given a space $V$ and configuration $\mathcal{U}$, we extend into a perpendicular space $V^{\text {ext }}$ and form a new configuration $\mathcal{U}^{\text {ext }}$ by extending vectors in the small orbit of $\mathcal{U}$. Imposing the $\vee$-conditions on the extended configuration then constrains the various objects, notably the constants $h_{a}$ associated with each covector.

Definition 5. Let $\mathcal{U}$ be $a \bigvee$-system.

(a) A small orbit $\vartheta_{s}$ of the $\bigvee$-system is a finite set of covectors such that

$$
w_{1}-w_{2} \in \mathcal{U}
$$

for all $w_{1}, w_{2} \in \vartheta_{s}$, with $w_{1} \neq \pm w_{2}$;

(b) An invariant small orbit consists of pairs $\left(w, h_{w}\right)$, where $w$ is a small orbit covector with associated multiplicity $h_{w}$, which satisfies the following additional conditions:

(i) $\sum_{w \in \vartheta_{s}} h_{w} w(z)^{2}=h_{s}(z, z)$

(ii) $\sum_{w \in \vartheta_{s}} h_{w} w(z)=0$

for all $z \in V$, where $(z, z)$ is the metric given by (5).

The first part of the definition is just an adaption of the concept of a small orbit for Weyl groups, ${ }^{7}$ and the adjective "orbit" reflects this origin. In applications, this set could be invariant under the action of the Weyl group (and hence a bone-fide orbit), but even if there is no such group, we keep this adjective. In the second part of the definition, the adjective "invariant" is used since if $\mathcal{U}$ is a Coxeter configuration (i.e., the root system of a Coxeter group, with multiplicities equal on each orbit), the two conditions in part (b) are, by basic properties of invariant theory, automatically satisfied.

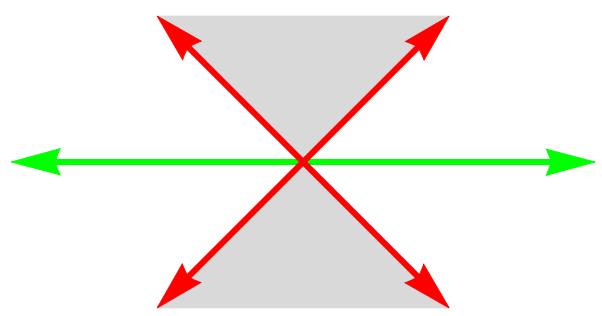

FIG. 2. The geometry of the $\bigvee$-configuration, rotated with the superimposed light-cone. 

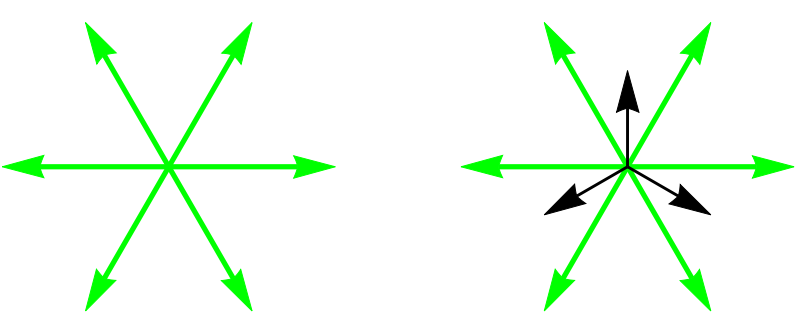

FIG. 3. The roots of $A_{2}$ and the small orbit superimposed on the roots.

Consider a Weyl group $W$ with $R$ being its irreducible root system and $P$ being its lattice of weights. Let $w_{i} \in P$ be a fundamental weight. Small orbits for such configurations were introduced and classified by Serganova.?

Theorem 6. Let $\omega_{i}$ be the fundamental weight vectors for a finite Coxeter group $W$. The small orbits of rank $\geq 2$ are given by the following:

1. $A_{n}: \vartheta_{s}=W \omega_{1}$ or $W \omega_{n}$

2. $B_{n}, B C_{n}, C_{n}(n \geq 4): \vartheta_{s}=W \omega_{1}$;

3. $B_{2}: \vartheta_{s}=W \omega_{1}$;

4. $\quad B_{3}: \vartheta_{s}=W \omega_{1}$ or $W \omega_{3}$;

5. $B C_{2}: \vartheta_{s}=W \omega_{1}$ or $2 W \omega_{2}$;

6. $C_{3}, B C_{3}: \vartheta_{s}=W \omega_{1}$;

7. $D_{n}(n \geq 3, n \neq 4): \vartheta_{s}=W \omega_{1}$

8. $D_{4} ; \vartheta=W \omega_{1}, W \omega_{3}$ or $W \omega_{4}$;

9. $G_{2}: \vartheta_{s}=W \omega_{1}$.

It is interesting to note that the $F_{4}, E_{6,7,8}$ Weyl groups do not have any small orbits. Since the constructions in this paper rely on the existence of such small orbits, the results here do not apply to these groups.

The main construction in the first part of this paper may be explained by the following example:

Example 7. We begin with the root system (automatically $a \bigvee$-system) for the Coxeter group $A_{2}$. This is shown in the left-hand side diagram in Fig. 3. The small orbit is given, by Theorem 6, by the orbit of a weight vector, and this orbit is shown, superimposed on the root system, in the right-hand side diagram in Fig. 3.

We now extend the configuration into a third dimension by adding a normal vector to the end of each small orbit vector and adding its negative. For $A_{2}$, this is shown in Fig. 4.

Repeating the construction gives an extended configuration. For $A_{2}$, this is shown in Fig. 5.

As in the previous example, the metric in the three-dimensional space, and in particular, its signature, depends on the geometry and multiplicities of these new extended vectors.

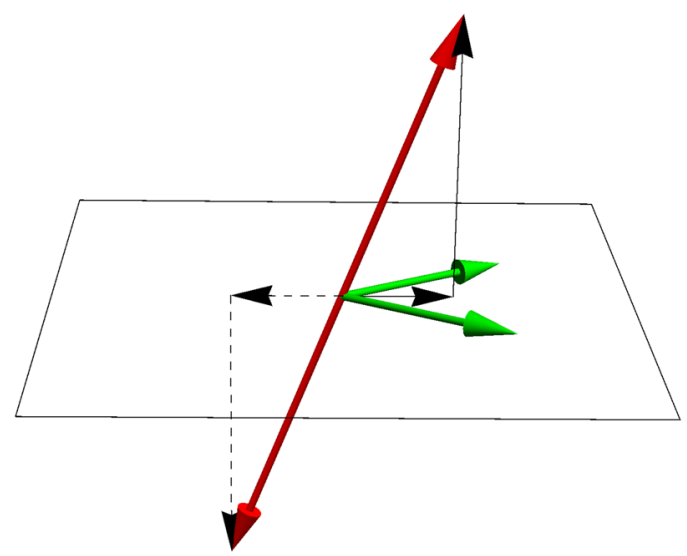

FIG. 4. The (partial) construction of an extended $\bigvee$-system. 


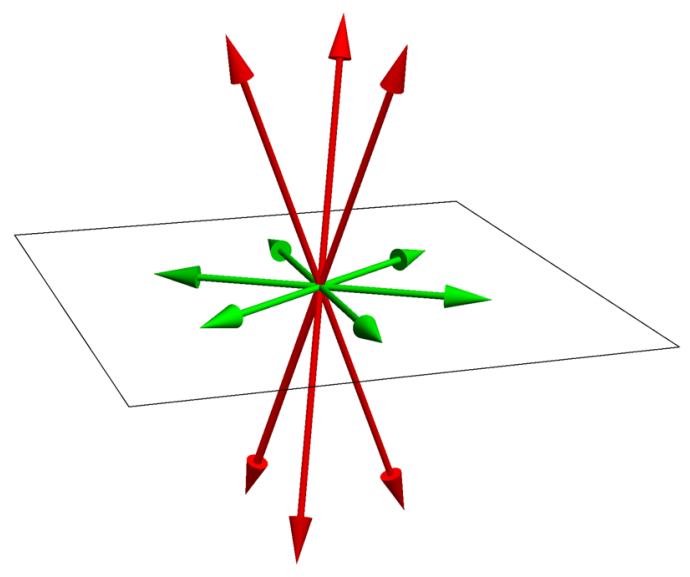

FIG. 5. An extended $\bigvee$-system.

This extended configuration is not, at present, a $\bigvee$-system. One now imposes the $\bigvee$-conditions to obtain algebraic conditions that restrict the new data. It is at this stage that the small orbit condition comes directly into play-it enables one to understand the two-dimensional configurations on each two-plane $\Pi$. These algebraic conditions are given in Lemma 9 and Theorem 14.

This construction has, so far, not resulted in any new $\bigvee$-systems. However, the results shed a new light on the geometry of the configurations and their origins, which enables the link with extended affine Weyl groups, their Frobenius and almost-dual Frobenius manifolds, and trigonometric $\bigvee$-systems to be made.

\section{EXTENDED V-SYSTEMS}

\section{A. Extended configurations}

We begin by extending $V$ by a one-dimensional space $V^{\perp}$,

$$
V^{e x t}=V \oplus V^{\perp}
$$

where $V^{\perp}=\operatorname{span}\left\{n^{\vee}\right\}$ and $V$ and $V^{\perp}$ are perpendicular subspaces of $V^{\text {ext }}$. The metric $(,)^{\text {ext }}$ on $V^{\text {ext }}$ is determined by the original metric on $V$ and the value $\left(n^{\vee}, n^{\vee}\right)^{\text {ext }}$ that defines the perpendicular scale. Thus,

$$
\left(z_{o}+z^{\perp}, z_{o}+z^{\perp}\right)^{e x t}=\left(z_{o}, z_{0}\right)+\left(z^{\perp}, z^{\perp}\right)^{e x t}, \quad z_{0} \in V, z^{\perp} \in V^{\perp} .
$$

With this metric, one can define the covector $n$ corresponding to $n^{\vee}$. Once this extended space has been defined, one can define the extended configuration $\mathcal{U}^{\text {ext }}$.

Definition 8. Let $\mathcal{U}$ be $a \bigvee$-system with an invariant small orbit $\vartheta_{s}$. The extended configurations $\mathcal{U}^{\text {ext }}$ are defined as

$$
\mathcal{U}^{\text {ext }}=\mathcal{U} \cup\left\{ \pm(w+n), w \in \mathcal{V}_{s}\right\} \cup\{ \pm n\} .
$$

We exclude reducible configurations, i.e., trivial extensions of the type $\mathcal{U} \cup\{ \pm n\}$. The corresponding multiplicities for the new covectors will be denoted $h_{w}$ [corresponding to the new covectors $\pm(w+n)$ ] and $h_{n}$ (corresponding to the new covectors $\pm n$ ).

We now have two metrics on $V^{\text {ext }}$, the canonical metric given by (5) (now summed over the extended configuration) and the orthogonal decomposition given by (6). The following lemma gives necessary and sufficient conditions for these to be equal:

Lemma 9. Let $\mathcal{U}$ be $a \bigvee$-system with an invariant small orbit $\vartheta_{s}$. The two metrics agree, i.e.,

$$
h_{\mathcal{U}^{\text {ext }}}(x, y)^{\text {ext }}=\sum_{\alpha \in \mathcal{U}^{\text {ext }}} h_{\alpha} \alpha(x) \alpha(y), \quad x, y \in V^{\text {ext }},
$$

if and only if

$$
h_{\mathcal{U}}+2 h_{\vartheta_{s}}=2\left\{h_{n}+\sum_{w \in \vartheta_{s}} h_{w}\right\}\left(n^{\vee}, n^{\vee}\right) .
$$


With this, $h_{\mathcal{U}^{\text {ext }}}=h_{\mathcal{U}}+2 h_{s}$.

Proof. We prove this in the case $x=y$; the full result then follows from polarization. Decomposing $z=z_{o}+z^{\perp}$ gives

$$
\begin{aligned}
\sum_{\alpha \in \mathcal{U}^{\text {ext }}} h_{\alpha} \alpha(z)^{2}= & \sum_{\alpha \in \mathcal{U}} h_{\alpha} \alpha(z)^{2}+\sum_{w \in \vartheta_{s}}\left(h_{w}[(w+n)(z)]^{2}+h_{w}[-(w+n)(z)]^{2}\right) \\
& +h_{n}[n(z)]^{2}+h_{n}[-n(z)]^{2} \\
= & \sum_{\alpha \in \mathcal{U}} h_{\alpha} \alpha\left(z_{o}\right)^{2}+2 \sum_{w \in \vartheta_{s}} h_{w}\left[w\left(z_{o}\right)+n\left(z^{\perp}\right)\right]^{2}+2 h_{n} n\left(z^{\perp}\right)^{2} \\
= & \left(h_{\mathcal{U}}+2 h_{s}\right)\left(z_{o}, z_{o}\right)+\left(2 h_{n}+\sum_{w \in \vartheta_{s}} h_{w}\right) n\left(z^{\perp}\right)^{2}
\end{aligned}
$$

where the invariant conditions have been used to derive the last line.

Since $\operatorname{dim} V^{\perp}=1, z^{\perp}=\mu n^{\vee}$ for some scalar $\mu$ and $n\left(z^{\perp}\right)=\left(n^{\vee}, z^{\perp}\right)^{\text {ext }}$,

$$
n\left(z^{\perp}\right)^{2}=\left(n^{\vee}, n^{\vee}\right)^{e x t}\left(z^{\perp}, z^{\perp}\right)^{e x t} .
$$

Thus,

$$
\sum_{\alpha \in \mathcal{U}^{\text {ext }}} h_{\alpha} \alpha(z)^{2}=\left(h_{\mathcal{U}}+2 h_{s}\right)\left(z_{0}, z_{o}\right)+\left(2 h_{n}+\sum_{w \in \vartheta_{s}} h_{w}\right)\left(n^{\vee}, n^{\vee}\right)^{e x t}\left(z^{\perp}, z^{\perp}\right)^{e x t}
$$

Since $(z, z)^{\text {ext }}=\left(z_{0}, z_{0}\right)+\left(z^{\perp}, z^{\perp}\right)^{\text {ext }}$, the result follows.

Example 10. Let $\mathcal{U}=\mathcal{R}_{A_{n}}$. We assume for now (these conditions will follow from the imposition of the $\bigvee$-conditions) that $h_{n}=0, h_{\alpha}=1$ for $\alpha \in \mathcal{R}_{A_{n}}$ (this fixes $h_{\mathcal{U}}=2(n+1)$, the (dual) Coxeter number of $A_{n}$ ), and $h_{w}=$ constant for $w \in \vartheta_{s}$. There are two (separate) small orbits-the orbit (under the $A_{n}$-group) of the highest and lowest weight vectors (the two families reflecting the symmetry of the Coxeter/Dynkin diagram). We first find the constant $h_{s}$. From symmetry/invariant theory, it follows that

$$
\begin{aligned}
\sum_{w \in \mathcal{\vartheta}_{s}} h_{w} w\left(z_{o}\right)^{2} & =h_{s}\left(z_{o}, z_{o}\right), \\
\sum_{w \in \mathcal{\vartheta}_{s}} h_{w} w\left(z_{o}\right) & =0
\end{aligned}
$$

for $z_{o} \in V$. Thus, the invariant conditions are automatically satisfied. To find $h_{s}$, we let $z_{o}=\alpha$ and sum over $\alpha \in \mathcal{R}_{A_{n}}$. Thus,

$$
h_{w} \sum_{w \in \vartheta_{s}} \sum_{\alpha \in \mathcal{R}_{A_{n}}}(w, \alpha)^{2}=h_{w} \sum_{\mathcal{R}_{A_{n}}}(\alpha, \alpha)
$$

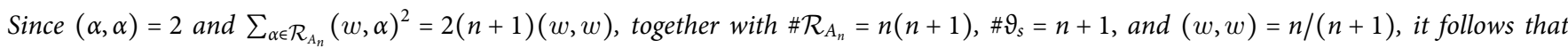
$h_{s}=h_{w}$. The number of elements in $\vartheta_{s}$ follows from Serganova's classification and standard properties of weight vectors.

Having found $h_{s}$, the result of Lemma 9 yields the condition

$$
\left(n^{\vee}, n^{\vee}\right)^{e x t}=\frac{1}{h_{w}}+\frac{1}{1+n} .
$$

Thus, the construction gives a one-parameter family of configurations, controlled by $h_{w}$ [or alternatively, controlled by the perpendicular scale-the length $\left.\left(n^{\vee}, n^{\vee}\right)\right]$.

Example 4 falls into this class, with $n=1, h_{w}=-1$. This gives $\left(n^{\vee}, n^{\vee}\right)^{\text {ext }}=-\frac{1}{2}$, reflecting the split signature of the metric.

\section{B. Imposition of the $\mathrm{V}$-conditions}

To impose the $\bigvee$-conditions on the extended configuration $\mathcal{U}^{\text {ext }}$, it is first necessary to classify the two-dimensional arrangements. Since $\mathcal{U}$ is a priori a $\bigvee$-system, one only has to understand plane arrangements through the origin and including some combination of the vectors $\pm(w+n), \pm n$. It is here that the small orbit condition comes into play. Given $w_{i}, w_{j} \in \vartheta_{s}$, consider the plane containing the vectors $w_{i}+n, w_{j}$ $+n$. Since

$$
w_{i}-w_{j} \in \operatorname{span}\left\{w_{i}+n, w_{j}+n\right\}
$$


it follows from the small orbit condition that the plane also contains an element $\alpha \in \mathcal{U}$. The set of planes for which one needs to impose the $\vee$ conditions depend on the geometric properties of the small orbit.

As is apparent from Fig. 3, unlike roots, the negative of a small orbit vector may, or may not, be a small orbit vector. If it is not, then the pure normal vectors in the extended configuration must be absent.

Lemma 11. Let $w \in \vartheta_{s}$, and suppose that no multiple of $w$ belongs to $\vartheta_{s}$. Then,

$$
\mathcal{U}^{\text {ext }}=\mathcal{U} \cup\left\{ \pm(w+n), w \in \mathcal{\vartheta}_{s}\right\} .
$$

Proof. Consider the intersection of the planes containing $\{ \pm n\}$ with $\mathcal{U}$. Such configurations take the form where the vectors $\pm(w+n)$ and/or $\pm n$ may, or may not, be present in the configuration:

- If $\pm(w+n)$ are not present, the remaining vectors are perpendicular and the $\bigvee$-conditions are vacuous.

- If $\pm(w+n)$ are present ( $s o h_{w} \neq 0$ ), the vectors $\pm \alpha$ may or may not be present. In either case, the $\bigvee$-conditions imply that $h_{w}=0$, and so such configurations cannot occur (it is here that the assumption $-w \notin \vartheta_{s}$ is used. Without this, additional terms could appear) (Fig. 6).

Thus, one arrives at a reducible configuration, so by the definition of $\mathcal{U}^{\text {ext }}$ (which excludes such reducible configurations),

$$
\mathcal{U}^{\text {ext }}=\mathcal{U} \cup\left\{ \pm(w+n), w \in \vartheta_{s}\right\}
$$

Since the small orbit vectors of $B_{n}$ have the property that $\pm w \in \vartheta_{s}$ and the small orbits vectors of $A_{n \geq 2}$ do not (as proved in Ref. 7), we define the following:

Definition 12. Let $w \in \vartheta_{s}$, and suppose that the only possible multiples of $w$ that belong to $\vartheta_{s}$ are $\pm w$.

(a) if $-w \notin \vartheta_{s}$, then $\mathcal{U}^{\text {ext }}$ is said to be of A-type;

(b) if $-w \in \vartheta_{s}$, then $\mathcal{U}^{\text {ext }}$ is said to be of B-type.

This gives a mechanism to "grow" root systems. For example, consider Fig. 5. By suitably fixing the perpendicular scale, this becomes the standard $A_{3}$ configuration.

Example 13.

- $\left(\mathcal{R}_{A_{n}}\right)^{\text {ext }}$ is of A-type;

- $\left(\mathcal{R}_{B_{n}}\right)^{\text {ext }}$ is of B-type;

In fact, with specific choices of the perpendicular direction scale,

$$
\begin{aligned}
& \left(\mathcal{R}_{A_{n}}\right)^{\text {ext }} \cong \mathcal{R}_{A_{n+1}}, \\
& \left(\mathcal{R}_{B_{n}}\right)^{\text {ext }} \cong \mathcal{R}_{B_{n+1}} .
\end{aligned}
$$

One now has to impose the $\bigvee$ conditions on the planes $\operatorname{span}\left\{w_{i}+n, w_{j}+n\right\}$ and, in particular, on vectors in the intersection span $\left\{w_{i}\right.$ $\left.+n, w_{j}+n\right\} \cap \mathcal{U}$. In general, one cannot say much about this intersection. It is at this stage that the small orbit property comes into play: it enables one to know what vectors are in this set. One obtains the two-plane configurations (see Fig. 7), and in the following theorem, the $\checkmark$-conditions are applied to these two-plane configurations.

Theorem 14. Consider a configuration of A-or B-type. The imposition of the $\bigvee$-conditions on the extended configurations $\mathcal{U}^{\text {ext }}$ results in the constraints on the data $\left\{h_{w_{i}}, h_{n}, n^{\vee}\right\}$,
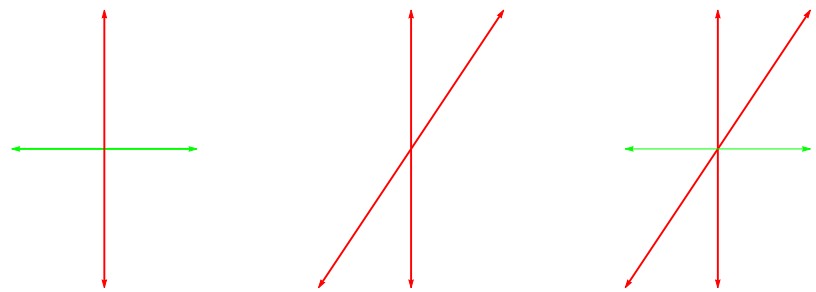

FIG. 6. Two-plane configuration including the normal direction. 

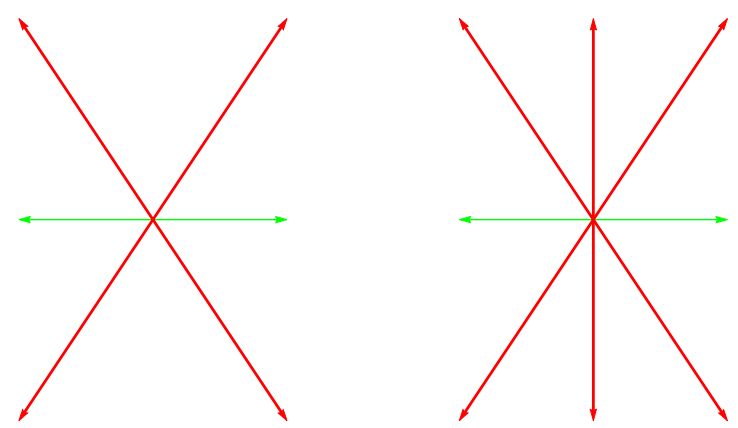

FIG. 7. Two-plane configurations.

$$
\begin{aligned}
h_{w_{i}}\left[\left(w_{i}^{\vee}, w_{i}^{\vee}\right)-\left(w_{i}^{\vee}, w_{j}^{\vee}\right)\right] & =h_{w_{j}}\left[\left(w_{j}^{\vee}, w_{j}^{\vee}\right)-\left(w_{i}^{\vee}, w_{j}^{\vee}\right)\right], \\
h_{w_{i}}\left[\left(w_{i}^{\vee}, w_{j}^{\vee}\right)+\left(n^{\vee}, n^{\vee}\right)\right] & =h_{\alpha}\left[\left(w_{j}^{\vee}, w_{j}^{\vee}\right)-\left(w_{i}^{\vee}, w_{j}^{\vee}\right)\right], \\
h_{w_{j}}\left[\left(w_{i}^{\vee}, w_{j}^{\vee}\right)+\left(n^{\vee}, n^{\vee}\right)\right] & =h_{\alpha}\left[\left(w_{i}^{\vee}, w_{i}^{\vee}\right)-\left(w_{i}^{\vee}, w_{j}^{\vee}\right)\right],
\end{aligned}
$$

where $\alpha=w_{j}-w_{i}$, and in addition (if the system is of B-type),

$$
h_{n}\left(n^{\vee}, n^{\vee}\right)=h_{\alpha}\left(w^{\vee}, w^{\vee}\right)+2 h_{w}\left\{\left(w^{\vee}, w^{\vee}\right)-\left(n^{\vee}, n^{\vee}\right)\right\} .
$$

The proof is by direct calculation, and details will not be given. It follows from applying Definition 3 to all two-plane configurations containing extended vectors: details may be found in Ref. 14. It is here that the small orbit geometry comes into play-it gives control over the vectors in $\Pi \cap \mathcal{U}^{\text {ext }}$. Note that this over-constrains the data $\left\{h_{w}, n^{\vee}\right\}$, but the constraints are completely determined by the geometry of small orbits.

Example 15. Let $\mathcal{U}=\mathcal{R}_{A_{n}}$. Then, from standard properties of weight vectors,

$$
\left(w_{i}^{\vee}, w_{j}^{\vee}\right)=\delta_{i j}-\frac{1}{n+1} .
$$

With these, one obtains $h_{w_{i}}=h_{w_{j}}$, i.e., all $h_{w_{i}}$ are equal, and, with the normalization $h_{\alpha}=1, \alpha \in \mathcal{U}$, the condition

$$
\left(n^{\vee}, n^{\vee}\right)=\frac{1}{h_{w}}+\frac{1}{1+n} .
$$

Note that this is the same condition that comes from Example 10. Thus, imposition of the $\bigvee$-conditions yields no new constraints.

Example 16. Let $\mathcal{U}=\mathcal{R}_{G_{2}}$. We normalize these roots-generated by simple roots $\alpha$ and $\beta$ by the conditions $(\alpha, \alpha)=2,(\alpha, \beta)=-3,(\beta, \beta)=6$. From (5), one finds

$$
6 h_{s}+18 h_{l}=h_{\mathcal{U}},
$$

where $h_{s}$ and $h_{l}$ are the multiplicities of the short and long roots. From Ref. 7, the small orbit is the $A_{2}$-subsystem generated by the set $\{ \pm \alpha, \pm(\alpha$ $+\beta), \pm(2 \alpha+\beta)\}$, and using this, one finds

$$
h_{\vartheta_{s}}=6 h_{w} .
$$

Lemma 9 implies

$$
3 h_{s}+9 h_{l}+6 h_{w}=\left(h_{n}+6 h_{w}\right)\left(n^{\vee}, n^{\vee}\right) .
$$

Theorem 14 then implies the equation

$$
\begin{aligned}
-3 h_{l}+h_{n}\left\{-1+\left(n^{\vee}, n^{\vee}\right)\right\} & =0, \\
-2 h_{s}-4 h_{w}+\left\{h_{n}+2 h_{w}\right\}\left(n^{\vee}, n^{\vee}\right) & =0 .
\end{aligned}
$$

Assuming $h_{w} \neq 0$ (otherwise the construction collapses), one can solve equations to obtain the extended configuration data in terms of the original $\vee$-data $\left\{h_{s}, h_{l}\right\}$, 


$$
\begin{aligned}
h_{w} & =\frac{1}{2}\left(h_{s}-3 h_{l}\right), \\
h_{n} & =\frac{3\left(h_{s}-3 h_{l}\right)^{2}}{h_{s}+3 h_{l}}, \\
\left(n^{\vee}, n^{\vee}\right) & =\frac{h_{s}+3 h_{l}}{h_{s}-3 h_{l}} .
\end{aligned}
$$

Note that one requires a slight constraint on the original data: $h_{s} \neq \pm 3 h_{l}$. This extended $\bigvee$-system coincides, after some linear algebra and redefinitions, to the system $G_{3}(t)$ presented in Ref. 15.

\section{GENERALIZED ROOT SYSTEMS AND THEIR EXTENSIONS}

The examples above have all been based on the system $\mathcal{U}$ being the root system of a (finite) Coxeter group. A wider class of examples come from the generalized root systems. Following Refs. 7 and 15, we have the following:

Definition 17. Let $V$ be a finite-dimensional complex vector space with a non-degenerate bilinear form (,). The finite set $\mathcal{U} \subset V \backslash\{0\}$ is called a generalized root system if the following conditions are fulfilled:

1. $\mathcal{U}$ spans $V$ and $\mathcal{U}=-\mathcal{U}$;

2. if $\alpha, \beta \in \mathcal{U}$ and $(\alpha, \alpha) \neq 0$, then $2 \frac{(\alpha, \beta)}{(\alpha, \alpha)} \in \mathbb{Z}$ and $r_{\alpha}(\beta)=\beta-2 \frac{(\alpha, \beta)}{(\alpha, \alpha)} \alpha \in \mathcal{U}$;

3. if $\alpha \in \mathcal{U}$ and $(\alpha, \alpha)=0$, then for any $\beta \in \mathcal{U}$ such that $(\alpha, \beta) \neq 0$, at least one of the vectors $\beta+\alpha$ or $\beta-\alpha$ belongs to $\mathcal{U}$.

The classification of irreducible generalized root systems was given in Ref. 7. In Secs. III A and III B, we analyze certain classes of such generalized root systems, constructing small orbits and hence extended configurations on which the $\bigvee$-conditions are imposed. Such configurations have much in common with the admissible deformations of the generalized root systems. ${ }^{16} \mathrm{We}$ also give, for the two classical series $A(m, n)$ and $B(m, n)$, an interpretation of a "reflection" in an isotropic root and a model for such an action. Roughly, one has two Coxeter groups that interchange-depending on the sign of $(\alpha, \alpha)$-the zeros and poles of a rational function and interchange a zero and a pole in the case of an isotropic root. Such an interpretation originates from the superpotential construction for almost-dual Frobenius manifolds.

\section{A. The series $A(m, n)$}

Consider the following data:

$$
\begin{aligned}
& \mathcal{U}=\left\{\alpha_{i j}:=e_{i}-e_{j}, i \neq j, \quad i, j=0, \ldots, n+m\right\}, \\
& \mathcal{E}_{i}= \begin{cases}+1 & \text { if } i=0, \ldots, m \\
-1 & \text { if } i=m+1, \ldots, n+m,\end{cases} \\
& g=\left.\sum_{i=0}^{m+n} \varepsilon_{i}\left(d z^{i}\right)^{2}\right|_{\sum \varepsilon_{j} j=0} .
\end{aligned}
$$

This constitutes a generalized root system (recall that the elements of $\mathcal{U}$ are covectors and the metric $g$ defines the vectors) and, as may be easily verified, a $\bigvee$-system with multiplicities $h_{\alpha_{i j}}=\varepsilon_{i} \varepsilon_{j}$. In particular, the metric $g$ takes the form of (5) with $h_{\mathcal{U}}=m+1-n$. Note

$$
\left(\alpha_{i j}^{\vee}, \alpha_{i j}^{\vee}\right)=\varepsilon_{i}+\varepsilon_{j},
$$

so the squared lengths can be $+2,0$ or -2 , reflecting the split signature of the metric $g$. If $n=0$, the system reduces to the standard Coxeter root system for $A_{m}$.

The small orbit is defined as follows: ${ }^{7}$

$$
\vartheta=\left\{w_{i}:=e_{i}+\frac{1}{n-m+1} \sum_{r=0}^{m+n} \varepsilon_{r} e_{r}\right\}
$$

(so trivially, $\alpha_{i j}=w_{i}-w_{j}$ ). That this is the only small orbit (up to sign) was proved in Ref. 7 .

Applying Theorem 14 gives the multiplicities $h_{w_{i}}=\varepsilon_{i} h_{w}$ for some $h_{w}$, and with this, the invariant conditions imply that $h_{\vartheta}=h_{w}$. Finally, one obtains the condition

$$
\frac{1}{h_{w}}+\frac{1}{m+1-n}=\left(n^{\vee}, n^{\vee}\right) \text {. }
$$

This then gives an extended $\bigvee$-system $\mathcal{U}^{\text {ext }}$, with one free parameter: $h_{w}$ fixes the perpendicular scale, or vice versa. 


\section{B. The series $B(m, n)$}

Consider the following data:

$$
\begin{aligned}
\mathcal{U} & =\left\{ \pm e_{i} \pm e_{j}, i \neq j, i, j=1, \ldots, n+m\right\} \cup\left\{e_{i}, i=1, \ldots, n+m\right\}, \\
\mathcal{E}_{i} & = \begin{cases}+1 & \text { if } i=1, \ldots, m \\
-1 & \text { if } i=m+1, \ldots, n+m,\end{cases} \\
g & =\sum_{i=1}^{m+n} \varepsilon_{i}\left(d z^{i}\right)^{2} .
\end{aligned}
$$

This constitutes a generalized root system (recall, elements of $\mathcal{U}$ are covectors and the metric $g$ defines the vectors) and, as may be easily verified, a $\bigvee$-system with multiplicities $h_{ \pm e_{i} \pm e_{j}}=h \varepsilon_{i} \varepsilon_{j}$ and $h_{ \pm e_{i}}=2 \varepsilon_{i}\left(2 \varepsilon_{i}+\gamma\right)$ with $\gamma$ arbitrary. Note

$$
\left(\alpha_{i j}^{\vee}, \alpha_{i j}^{\vee}\right)=\varepsilon_{i}+\varepsilon_{j}, \quad\left(\alpha_{i}^{\vee}, \alpha_{i}^{\vee}\right)=\varepsilon_{i}
$$

(where $\alpha_{i j}= \pm e_{i} \pm e_{j}$ and $\alpha_{i}= \pm e_{i}$ ), so the squared lengths can be $+2,+1,0,-1$, or -2 , reflecting the split signature of the metric $g$. If $n=0$, the system reduces to the standard Coxeter root system for $B_{m}$.

The small orbit is defined as follows:

$$
\vartheta=\left\{ \pm e_{i}\right\}
$$

That this is the only small orbit (up to sign) was proved in Ref. 7.

Applying Lemma 9 and Theorem 14 gives the multiplicities $h_{w_{i}}=\varepsilon_{i} h_{w}$ and the conditions

$$
\begin{aligned}
h_{w} & =\frac{h}{\left(n^{\vee}, n^{\vee}\right)}, \\
h_{n} & =\frac{2 h}{\left(n^{\vee}, n^{\vee}\right)}+\frac{h \gamma}{\left(n^{\vee}, n^{\vee}\right)} .
\end{aligned}
$$

This then gives an extended $\bigvee$-system $\mathcal{U}^{\text {ext }}$, with one free parameter: the perpendicular scale.

\section{Symmetries of the extended configurations}

Consider again the extended configuration shown in Fig. 5. The original configuration $\mathcal{U}=R_{A_{2}}$ is by definition invariant under reflections-the group $A_{2}$-as are the small orbit vectors (which form an irregular orbit). The reflection generated by these roots can be extended to the whole space (acting trivially in the perpendicular direction). Thus, the whole three-dimensional configuration is invariant under $A_{2}$ but not, in general, under reflections generated by the new roots $\pm(w+n)$. Clearly, this idea generalizes-any symmetry is inherited by the extended configuration.

However, we now have to consider the generalized root system of $A(m, n)$, which has roots of positive, negative, and zero length. The non-isotropic roots define reflections in the hyperplanes $\alpha_{i j}(z)=0$, and the whole configuration $\mathcal{U}^{\text {ext }}$ is invariant under reflections in the original roots.

More explicitly, if $\alpha_{i j}$ is a non-isotropic root, then it is easy to show, using the small orbit property, that for $w_{i, j, k} \in \mathcal{\vartheta}_{s}$,

$$
r_{\alpha_{i j}}\left(w_{k}\right)= \begin{cases}w_{k} & \text { if } \mathrm{i}, \mathrm{j}, \mathrm{k}, \text { distinct } \\ w_{i} & \text { if } j=k \\ w_{j} & \text { if } i=k,\end{cases}
$$

(on writing $\alpha_{r s}=w_{r}-w_{s}$ ) and the reflection $r_{\alpha_{i j}}\left(\alpha_{r s}\right)$ can then be found using linearity. However, as an abstract group, one may define a transformation $r_{\alpha_{i j}}$ for all roots (including isotropic roots) by the above formulas. While $\left(r_{\alpha_{i j}}\right)^{2}=i d$, the lengths of the covectors are not preserved under the action of $r_{\alpha_{i j}}$. For example, since

$$
\left(w_{i}^{\vee}, w_{j}^{\vee}\right)=\varepsilon_{i} \delta_{i j}+\frac{1}{n-(m+1)}
$$

a "reflection" in an isotropic root will change the length of the roots. With this interpretation/definition of $r_{\alpha_{i j}}$, the whole extended configuration $\mathcal{U}^{\text {ext }}$ is invariant under the above action of $r_{\alpha_{i j}}$. 
If one extends the action of $r_{\alpha_{i j}}$ to the multiplicities $\varepsilon_{i}$ via the formula

$$
r_{\alpha_{i j}}\left(\varepsilon_{k}\right)= \begin{cases}\varepsilon_{k} & \text { if } i, j, k \text {, distinct } \\ \varepsilon_{i} & \text { if } j=k \\ \varepsilon_{j} & \text { if } i=k,\end{cases}
$$

then the plane $\sum \varepsilon_{i} z^{i}=0$ is invariant under the action of $r_{\alpha_{i j}}$ (which now interchanges both $z^{i}$ with $z^{j}$ and $\varepsilon_{i}$ with $\varepsilon_{j}$ ).

A model for this comes from the space of rational functions,

$$
\lambda\left(\left\{z^{k}\right\},\left\{\varepsilon_{k}\right\}: z\right)=\left.\prod_{k=0}^{m+n}\left(z-z^{k}\right)^{\varepsilon_{k}}\right|_{\sum \varepsilon_{j} j=0} .
$$

Hence, for all roots $\alpha_{i j}$,

$$
\lambda\left(\left\{r_{\alpha_{i j}} z^{i}\right\},\left\{r_{\alpha_{i j}} \varepsilon_{i}\right\}: z\right)=\lambda\left(\left\{z^{i}\right\},\left\{\varepsilon_{i}\right\}: z\right) .
$$

If $n=0$, then one recovers the standard invariant polynomial model for $A_{m}$,

$$
\lambda(z)=\left.\prod_{i=0}^{m}\left(z-z^{i}\right)\right|_{\sum z^{r}=0},
$$

which is invariant under the interchange of zeros. Repeating the argument for $B(m, n)$ results in the rational function

$$
\lambda\left(\left\{z^{k}\right\},\left\{\varepsilon_{k}\right\}: z\right)=\prod_{k=1}^{m+n}\left(z^{2}-\left(z^{k}\right)^{2}\right)^{\varepsilon_{k}} .
$$

If $n=0$, then one recovers the standard invariant polynomial model for $B_{m}$.

\section{LEGENDRE TRANSFORMATIONS AND TRIGONOMETRIC V-SYSTEMS}

A symmetry of the WDVV equations are transformations

$$
\begin{aligned}
z^{\alpha} & \mapsto \hat{z}^{\alpha}, \\
g_{\alpha \beta} & \mapsto \hat{g}_{\alpha \beta}, \\
F & \mapsto \hat{F}
\end{aligned}
$$

that preserves the equations. Here, $\left\{z^{\alpha}\right\}$ are the flat coordinates of the metric $g$. In Ref. 8, Legendre-type transformations were constructed. These take the form of the transformations

$$
\begin{aligned}
\hat{z}_{\alpha} & =\partial_{z^{\alpha}} \partial_{z^{\kappa}} F, \\
\frac{\partial^{2} \hat{F}}{\partial \hat{z}^{\alpha} \hat{z}^{\beta}} & =\frac{\partial^{2} F}{\partial z^{\alpha} \partial z^{\beta}}, \\
g_{\alpha \beta} & =\hat{g}_{\alpha \beta} .
\end{aligned}
$$

Such transformations are generated by a constant vector field $\partial=\frac{\partial}{\partial z^{\kappa}}$, where $\kappa=1, \ldots, n$.

In this section, we apply such a Legendre transformation to the solution of the WDVV equations given by extended $\vee$-systems, as constructed above. Such systems have a distinguished vector that may be used to define the Legendre transformation, namely, the vector in the perpendicular, or extended, direction $\partial=\frac{\partial}{\partial z^{\perp}}$.

Since $\mathcal{U}^{\text {ext }}=\mathcal{U} \cup \mathcal{U}^{\prime}$, where $\mathcal{U}$ is the original, unextended $\vee$-system, the new variables are given purely in terms of the vector in $\mathcal{U}^{\prime}$ and hence only on the small orbit data. In particular,

$$
\hat{z}_{\alpha}=\frac{\partial^{2}}{\partial z^{\alpha} \partial z^{\perp}}\left\{\sum_{\beta \in \mathcal{U}^{\prime}} h_{\beta} \beta(z)^{2} \log \beta(z)\right\}
$$

since terms involving $\alpha(z)$ with $\alpha \in \mathcal{U}$ are $z^{\perp}$ independent. The difficulty in applying such a transformation is computational: one has to invert the above change of variables. In the following example, we invert these equations for the case when $\mathcal{U}=\mathcal{R}_{A_{n}}$. The procedure is very general and may easily be applied to other systems. 
Example 18. We study the case when $\mathcal{U}=\mathcal{R}_{A_{n}}$ when the original system is the set of roots of the $A_{n}$ Coxeter group. To do this, we utilize the fact that for $A_{n}$, we have $\# \vartheta_{s}=n+1$, so we can use $n$ of them as a basis for $V$. We label these covectors $w_{i}, i=0, \ldots, n$ with $w_{0}=-\sum_{i=1}^{n} w_{i}$.

Using this basis, we define (recall $z=z_{o}+z^{\perp}$ )

$$
\begin{aligned}
z_{i} & =w_{i}(z), \quad i=0, \ldots, n, \\
z_{\perp} & =n(z) .
\end{aligned}
$$

Note that $\sum_{i=0}^{n} z_{i}=0$. With this, the change of variables given by (7) reduces to

$$
\begin{aligned}
& \hat{z}_{i}=\frac{\partial^{2}}{\partial z_{i} \partial z_{\perp}}\left\{\sum_{r=0}^{n} 2 h_{\beta}\left(z_{i}+z_{\perp}\right)^{2} \log \left(z_{i}+z_{\perp}\right)\right\}, \\
& \hat{z}_{\perp}=\frac{\partial^{2}}{\partial\left(z_{\perp}\right)^{2}}\left\{\sum_{r=0}^{n} 2 h_{\beta}\left(z_{i}+z_{\perp}\right)^{2} \log \left(z_{i}+z_{\perp}\right)\right\} .
\end{aligned}
$$

On absorbing constants or using the quadratic freedom in the definition of $F$, one obtains the simple system

$$
\begin{aligned}
& \hat{z}_{i}=4 h_{\beta}\left\{\log \left(z_{i}+z_{\perp}\right)-\log \left(z_{0}+z_{\perp}\right)\right\}, \\
& \hat{z}_{\perp}=4 h_{\beta} \sum_{r=0}^{n} \log \left(z_{i}+z_{\perp}\right),
\end{aligned}
$$

which is straightforward to invert. This yields

$$
\begin{aligned}
& z_{i}+z_{\perp}=e^{\frac{1}{4 h_{\beta}(n+1)}\left(\hat{z}_{0}+\hat{z}_{\perp}\right)} \cdot e^{\frac{1}{4 h_{\beta}} \hat{z}_{i}}, \quad i=1, \ldots, n, \\
& z_{0}+z_{\perp}=e^{\frac{1}{4 h_{\beta}(n+1)}\left(\hat{z}_{0}+\hat{z}_{\perp}\right)} .
\end{aligned}
$$

One could go further and solve these, but this is not actually required. All that is required are the terms $\alpha(z)$ and $\beta(z)$. In fact, the above formulas are precisely the $\beta(z)$-terms, and to find the $\alpha(z)$-terms, one can again use the small orbit property and write each $\alpha$ as the difference of two small orbit covectors. Thus, if $\alpha=w_{i}-w_{j}$, then

$$
\begin{aligned}
& \alpha(z)=\left(w_{i}-w_{j}\right)(z) \\
& = \begin{cases}z_{i}-z_{j} & \text { if } i, j \neq 0 \\
z_{0}-z_{j} & \text { if } i=0, j \neq 0\end{cases} \\
& = \begin{cases}e^{\frac{1}{4 h_{\beta}(n+1)}\left(\hat{z}_{0}+\hat{z}_{\perp}\right)}\left(e^{\frac{1}{4 h_{\beta}} \hat{z}_{i}}-e^{\frac{1}{4 h_{\beta}} \hat{z}_{j}}\right), & \text { if } i, j \neq 0 \\
e^{\frac{1}{4 h_{\beta}(n+1)}\left(\hat{z}_{0}+\hat{z}_{\perp}\right)}\left(1-e^{\frac{1}{4 h_{\beta}} \hat{z}_{i}}\right), & \text { if } i=0, j \neq 0 .\end{cases}
\end{aligned}
$$

To complete the Legendre transformation, one has to integrate the equations to find $\hat{F}$. Using these formulas, one finds, schematically, that

$$
\frac{\partial^{2} \hat{F}}{\partial \hat{z}_{i} \partial \hat{z}_{j}}=\text { linear term }+\sum_{\sigma} \text { terms involving }\left\{\log \left[1-e^{\sigma(\hat{z})}\right]\right\}
$$

(where the $\sigma$ are certain covectors-linear combinations of the original covectors) with similar formulas for the $\hat{z}_{\perp}$-derivatives. It is important to note that $\hat{z}_{\perp}$ only occurs in the linear terms in these expressions. Integrating yields a solution of the WDVV equations of trigonometric type,

$$
\hat{F}=\text { cubic term }+\sum_{\sigma \in \widehat{\mathcal{U}}} c_{\sigma} L i_{3}\left(e^{\sigma(z)}\right),
$$

where, again, $\hat{z}_{\perp}$ only occurs in the cubic term.

Explicit examples of this kind may be found in Refs. 9 and 13.

Example 19. Applying this Legendre transformation to Example 4 yields the prepotential ${ }^{13}$

$$
\hat{F}=\frac{1}{24} z_{\perp}^{3}-\frac{1}{8} z_{\perp} z^{2}+\frac{1}{2}\left\{L i_{3}\left(e^{z}\right)+L i_{3}\left(e^{-z}\right)\right\} .
$$


This is the almost-dual prepotential associated with the prepotential

$$
F=\frac{1}{2} t_{1}^{2} t_{2}+e^{t_{2}}
$$

which is constructed from the extended affine Weyl group $A_{1}^{(1)}$.

The above example shows a connection between extended $\bigvee$-systems and extended affine Weyl groups. This link-via a Legendre transformation-will form the subject of Sec. V.

\section{EXTENDED $\bigvee$-SYSTEMS AND ALMOST-DUALITY FOR EXTENDED AFFINE WEYL ORBIT SPACES}

In the early 1990s, two classes of solutions to the WDVV equations were constructed from a finite Coxeter group $W$. On the orbit space $\mathbb{C}^{N} / W$, a polynomial solution was found ${ }^{8}$ using the Saito construction. ${ }^{17}$ However, motivated by the appearance of the WDVV equation in Seiberg-Witten theory, solutions of the form

$$
F^{\star}=\frac{1}{4} \sum_{\alpha \in R_{W}} \alpha(z)^{2} \log \alpha(z)
$$

had also been found. Later in the decade, the link between these two classes of solutions was found, via the notion of almost-duality. ${ }^{2}$

Given a Frobenius manifold $F=\{\mathcal{M}, \circ, e, E, \eta\}$ (we denote the manifold and the corresponding prepotential by the symbol $F$ ), one may obtain a so-called almost-dual Frobenius manifold $F^{\star}=\{\mathcal{M} \backslash \mathcal{D}, \star, E, g\}$ by defining a new metric and multiplication by the formulas

$$
\begin{aligned}
g(X, Y) & =\eta\left(E^{-1} \circ X, Y\right), \\
X \star Y & =E^{-1} \circ X \circ Y .
\end{aligned}
$$

This new metric turns out to the flat coordinate (by virtue of the Frobenius manifold axioms) and compatible with the new multiplication. Using these facts, Dubrovin showed that the $\star$-multiplication provides us with a solution of the WDVV equations written in the flat coordinates of the new metric $g$.

Thus, given a Frobenius manifold $F$, we have an almost-dual manifold $F^{\star}$. However, one may apply a Legendre transformation to $F$ to get a new manifold $\hat{F}$ and apply almost duality to that. This is summarized in the diagram

$$
\begin{array}{ccc}
F & \stackrel{S_{\kappa}}{\longrightarrow} & \hat{F} \\
\downarrow & & \\
F^{\star} & & \\
& & \hat{F}^{\star}
\end{array}
$$

In Ref. 13, a twisted Legendre transformation was constructed,

$$
F^{\star} \stackrel{\hat{S}_{\kappa}}{\rightarrow} \hat{F}^{\star}
$$

The vector field that generates this twisted Legendre transformation is

$$
\tilde{\partial}=E \circ \partial,
$$

i.e., the generating field $\partial$ twisted by multiplication by the Euler vector field.

One of the main problems in the theory of almost-dual type solutions to the WDVV equations (this class including $\bigvee$-systems) is to indentify those which are precisely the almost-dual prepotentials to some Frobenius manifold. In Ref. 2, a reconstruction theorem was proved, but the theorem is extremely difficult to use in practice. It relies on solving a linear Lax pair and finding a vector field (to play the role of $e$ ) that acts on this solution in a specific way. In this section, we bypass this reconstruction theorem and prove the following theorem:

Theorem 20. Let $W$ be a finite irreducible classical Coxeter group of rank $l$, and let $\widetilde{W}$ be the extended affine Weyl group of $W$ with an arbitrary marked node. Then, up to a Legendre transformation, the almost-dual prepotentials of the classical extended affine Weyl group orbit spaces $\mathbb{C}^{l+1} / \widetilde{W}$ are, for specific values of the free data, the extended $\bigvee$-systems of the $\bigvee$-system $R_{W}$.

In particular:

- The almost-dual prepotential corresponding to the orbit space

$$
\mathbb{C}^{l+1} / \widetilde{W}^{(k)}\left(A_{l}\right)
$$

is the Legendre transformation, along the extended direction, of the extended $\vee$-system with 
(i) original $\bigvee$-system

$$
\begin{aligned}
\mathcal{U} & =R_{A_{l}}, \\
h_{\alpha} & =1 \quad \forall \alpha \in \mathcal{U} ;
\end{aligned}
$$

(ii) data for extension (of A-type)

$$
\begin{aligned}
\vartheta_{s} & =\left\{w\left(\omega_{1}\right) \mid w \in W\left(A_{l}\right)\right\}, \\
h_{w} & =-(l+1-k) \quad \forall w \in \mathcal{U}^{\text {ext }} ;
\end{aligned}
$$

(iii) superpotential data: The superpotential for the extended $\bigvee$-system is given by (2) with

$$
\mathbf{k}^{\text {ext }}=\{-(l+1-k), \underbrace{1, \ldots, 1}_{l+1}\} .
$$

- The almost-dual prepotential corresponding to the orbit space

$$
\mathbb{C}^{l+1} / \widetilde{W}^{(k)}\left(C_{l}\right)
$$

with a flat structure defined by the constant $m$, where $0 \leq m \leq l-k$, is the Legendre transformation, along the extended direction, of the extended $\bigvee$-system with [where $s=-2(l-(k+m)]$ :

(i) original $\bigvee$-system

$$
\begin{aligned}
\mathcal{U} & =R_{B_{l}}, \\
h_{\alpha_{\text {short }}} & =1+s, \\
h_{\alpha_{\text {long }}} & =1
\end{aligned}
$$

for all $\alpha_{\text {short/long }} \in \mathcal{U}$;

(ii) data for extension (of B-type)

$$
\begin{aligned}
\vartheta_{s} & =\left\{w\left(\omega_{1}\right) \mid w \in W\left(B_{l}\right)\right\}, \\
h_{w} & =-2 k \quad \forall w \in \mathcal{U}^{\text {ext }}, \\
h_{n} & =-2 k(s+2 k) ;
\end{aligned}
$$

(iii) superpotential data: The superpotential for the extended $\bigvee$-system is given by (3) with

$$
\mathbf{k}^{e x t}=\{-2 k, \underbrace{1, \ldots, 1}_{l}\} .
$$

The proof will utilize a Hurwitz space construction. For extended affine Weyl groups of type A, this construction was given in Ref. 11 . For types $B, C, D$, this was given in Ref. 12 .

Hurwitz spaces are moduli spaces of pairs $(\mathcal{C}, \lambda)$, where $\mathcal{C}$ is a Riemann surface of degree $g$ and $\lambda$ is a meromorphic function on $\mathcal{C}$ of degree $N$. It was shown in Ref. 8 that such spaces may be endowed with the structure of a Frobenius manifold. The $g=0$ case is particularly simple-meromorphic functions from the Riemann sphere to itself are just given by rational functions. It is into this category of Frobenius manifolds that the examples constructed above fall.

More specifically, the Hurwitz space $H_{g, N}\left(k_{1}, \ldots, k_{l}\right)$ is the space of equivalence classes $\left[\lambda: \mathcal{C} \rightarrow \mathbb{P}^{1}\right]$ of $N$-fold branched covers (Dubrovin uses the different notation $\left.H_{g, k_{1}-1, \ldots, k_{l}-1}\right)$ with

- $M$ simple ramification points $P_{1}, \ldots, P_{M} \in \mathcal{L}$ with distinct finite images $l_{1}, \ldots, l_{M} \in \mathbb{C} \subset \mathbb{P}^{1}$ and

- the preimage $\lambda^{-1}(\infty)$ consists of $l$ points $\lambda^{-1}(\infty)=\left\{\infty_{1}, \ldots, \infty_{l}\right\}$, and the ramification index of the map $p$ at the point $\infty_{j}$ is $k_{j}$ $\left(1 \leq k_{j} \leq N\right)$.

The Riemann-Hurwitz formula implies that the dimension of this space is $M=2 g+l+N-2$. One has also the equality $k_{1}+\cdots+k_{l}=N$. For $g>0$, one has to introduce a covering space, but this is unnecessary in the $g=0$ case that will be considered here.

In this construction, there is a certain ambiguity; one has to choose a so-called primary differential (also known as a primitive form). Different choices produce different solutions to the WDVV equations, but such solutions are related by the Legendre transformation $S_{\kappa}$. The 
Hurwitz data $\{\lambda, \omega\}$ from which one constructs a solution $F_{\{\lambda, \omega\}}$ consist of the map $\lambda$ (also known as the superpotential) and a particular primary differential $\omega .{ }^{8}$ Thus, again schematically, one has

$$
F_{\{\lambda, \omega\}} \stackrel{S_{\kappa}}{\longleftrightarrow} \hat{F}_{\{\lambda, \hat{\omega}\}}
$$

(note that the map $\lambda$ does not change, though it might undergo a coordinate transformation). The metrics $\langle\rangle,,($,$) and multiplications \circ, \star$ are determined by calculating certain residues at the critical points of the map $\lambda$.

Theorem 21.

$$
\begin{aligned}
& \left\langle\partial^{\prime}, \partial^{\prime \prime}\right\rangle=-\sum \underset{d \lambda=0}{\operatorname{res}}\left\{\frac{\partial^{\prime}(\lambda(v)) \partial^{\prime \prime} \lambda(v)}{\lambda^{\prime}(v)} \omega\right\}, \\
& \left\langle\partial^{\prime} \circ \partial^{\prime \prime}, \partial^{\prime \prime \prime}\right\rangle=-\sum \underset{d \lambda=0}{\operatorname{res}}\left\{\frac{\partial^{\prime} \lambda(v) \partial^{\prime \prime} \lambda(v) \partial^{\prime \prime \prime} \lambda(v)}{\lambda^{\prime}(v)} \omega\right\}, \\
& \left(\partial^{\prime}, \partial^{\prime \prime}\right)=-\sum \underset{d \lambda=0}{\operatorname{res}}\left\{\frac{\partial^{\prime} \log \lambda(v) \partial^{\prime \prime} \log \lambda(v)}{(\log \lambda)^{\prime}(v)} \omega\right\}, \\
& \left(\partial^{\prime} \star \partial^{\prime \prime}, \partial^{\prime \prime \prime}\right)=-\sum \underset{d \lambda=0}{\operatorname{res}}\left\{\frac{\partial^{\prime} \log \lambda(v) \partial^{\prime \prime} \log \lambda(v) \partial^{\prime \prime \prime} \log \lambda(v)}{(\log \lambda)^{\prime}(v)} \omega\right\} .
\end{aligned}
$$

Here, $\omega$ is the primary differential.

We divide the proof into the $A$ case and the $B, C, D$ cases.

\section{A. Extended affine Weyl orbit spaces of type $A$}

In Ref. 11, it was shown, given an extended affine group $\widetilde{W}^{(k)}\left(A_{l}\right)$, that the orbit space $\mathbb{C}^{l+1} / \widetilde{W}^{(k)}\left(A_{l}\right)$ maybe endowed with the structure of a Frobenius manifold. Additionally, it was shown that this space is isomorphic to the space of trigonometric polynomials of bi-degree $(k, l+1-k)$, namely, functions of the form

$$
\lambda(\tilde{z})=e^{i k \tilde{z}}+a_{1} e^{i(k-1) \tilde{z}}+\cdots+a_{l+1} e^{-i(l+1-k) \tilde{z}},
$$

with the Frobenius manifold structures being given by Theorem 21 with the choice of primary differential $\omega=d \tilde{z}$.

This space is related, via a Legendre transformation, to the Hurwitz space $\mathcal{M}_{k, l+1-k}$ of rational functions of the form

$$
\lambda(z)=z^{k}+\alpha_{1} z^{k-2}+\cdots+\alpha_{k-2} z+\alpha_{k-1}+\frac{\alpha_{k}}{z-z^{\circ}}+\cdots+\frac{\alpha_{l}}{\left(z-z^{\circ}\right)^{l+1-k}},
$$

with coordinates $\alpha_{i}, i=1, \ldots, l$ and $z^{\circ}$. Using the primary differential $\omega=d z$, one can show that the coefficient $\alpha_{k}$, by evaluating certain residues, is a flat coordinate, which we denote by $t^{\circ}$ (i.e., $\beta_{k}=t^{\circ}$ ). With this, one may define a change of primary differential

$$
d \tilde{z}=\left\{\partial_{t}{ }^{\circ} \lambda(z)\right\} d z
$$

so $\tilde{z}=\log (z-z)^{\circ}$, and this induces the Legendre transformation between the two Frobenius manifolds, i.e., this change of primary differential induces a change of variable that maps (8) to (9). Thus, the Frobenius manifold structures on $\mathbb{C}^{l+1} / \widetilde{W}^{(k)}\left(A_{l}\right)$ and $\mathcal{M}_{k, l+1-k}$ are related by a Legendre transformation.

Rewriting the rational function (9) in this form

$$
\lambda(z)=\left.\frac{\prod_{i=1}^{l+1}\left(z-z_{i}\right)}{\left(z-z^{\circ}\right)^{l+1-k}}\right|_{\sum_{i=1}^{l+1} z_{i}-(l+1-k) z^{\circ}=0}
$$

thus gives a $\bigvee$-system that is of extended type, i.e., an extension of the $A_{l} \bigvee$-system constructed above, with the zeros of the superpotential being flat coordinates for the metric $g$. This is a special case, with

$$
\mathbf{k}=\{-(l+1-k), \underbrace{1, \ldots, 1}_{l+1}\}
$$

of the general superpotential (2). 
At this stage, one could directly calculate the almost-dual prepotential from the trigonometric superpotential, and the result is a solution that is derived from a trigonometric $\bigvee$-system. ${ }^{13}$ However, we will take a different approach and perform the twisted Legendre transformation,

$$
\begin{array}{ccc}
\mathcal{M}_{k, l+1-k} & \stackrel{\text { Legendretransformation }}{\longleftrightarrow} & \mathbb{C}^{l+1} / \widetilde{W}^{(k)}\left(A_{l}\right) \\
\downarrow & \downarrow \\
\left\{\begin{array}{c}
\text { almost dual } \\
\text { prepotential of } \\
\text { rational type }
\end{array}\right\} \stackrel{\text { twistedLegendretransformation }}{\longleftrightarrow}\left\{\begin{array}{c}
\text { almost dual } \\
\text { prepotential of } \\
\text { trigonometric type }
\end{array}\right\}
\end{array}
$$

It turns out that with the choice $\partial=\partial_{t^{\circ}}$, the twisted Legendre field is actually constant.

Lemma 22. The twisted Legendre field $E \circ \frac{\partial}{\partial t^{\circ}}$ is constant in the $\left\{z^{i}\right\}$-variables. Moreover, the field is perpendicular to the space

$$
T V=\operatorname{span}\left\{\frac{\partial}{\partial w^{i}}, i=1, \ldots, l\right\}
$$

where the variables $z^{i}$ and $w^{i}$ are related by the linear change of variables,

$$
\begin{aligned}
w^{i} & =z^{i}-\frac{1}{l+1} \sum_{j=1}^{l+1} z^{j}, \quad j=1, \ldots, l+1, \\
w^{\perp} & =\frac{1}{l+1-k} \sum_{j=1}^{l+1} z^{j},
\end{aligned}
$$

together with the constraint $\sum_{i=1}^{l+1} w^{i}=0$.

Proof. We calculate

$$
g\left(E \circ \frac{\partial}{\partial t^{\circ}}, \frac{\partial}{\partial z^{i}}\right) .
$$

Using the above formulas,

$$
\begin{aligned}
g\left(E \circ \frac{\partial}{\partial t^{\circ}}, \frac{\partial}{\partial z^{i}}\right) & =\eta\left(\frac{\partial}{\partial t^{\circ}}, \frac{\partial}{\partial z^{i}}\right) \\
& =\sum \operatorname{res}_{d \lambda=0}\left\{\frac{\frac{\partial \lambda}{\partial t^{\circ}} \frac{\partial \lambda}{\partial z^{i}}}{\lambda^{\prime}} d z\right\} \\
& =\sum \operatorname{res}_{d \lambda=0}\left\{\frac{1}{\left(z-z^{\circ}\right)} \frac{\partial \lambda}{\partial z^{i}} \frac{1}{\lambda^{\prime}} d z\right\} .
\end{aligned}
$$

A standard residue calculation gives

$$
g\left(E \circ \frac{\partial}{\partial t^{\circ}}, \frac{\partial}{\partial z^{i}}\right)=-\frac{1}{l+1-k} .
$$

In these coordinates,

$$
g=\sum_{i=1}^{l+1}\left(d z^{i}\right)^{2}-\frac{1}{l+1-k}\left(\sum_{j=1}^{l+1} d z^{j}\right)^{2}
$$

and hence,

$$
E \circ \frac{\partial}{\partial t^{\circ}}=\frac{1}{k} \sum_{j=1}^{l+1} \frac{\partial}{\partial z^{j}} .
$$

In the $w^{i}$ variables,

$$
E \circ \frac{\partial}{\partial t^{\circ}}=-\frac{l+1}{k(l+i-k)} \frac{\partial}{\partial w^{\perp}}
$$


and

$$
g=\left.\sum_{i=1}^{l}\left(d w^{i}\right)^{2}\right|_{\sum w^{i}=0}-\frac{k(l+1-k)}{l+1}\left(d w^{\perp}\right)^{2}
$$

and hence, the twisted Legendre field is perpendicular to the space $T V$.

\section{B. Extended affine Weyl orbit spaces of type $B, C, D$}

In a similar way, given an extended affine Weyl group of type $B, C, D$, there exist Frobenius manifold structures on the corresponding orbit space. In Ref. 11, this was constructed for a specific choice of marked node, and in Ref. 12, this construction was generalized to the case of an arbitrary marked node. Thus, given a extended affine Weyl goup of $C$-type, $\widetilde{W}^{(k)}\left(C_{l}\right)$ one can construct a Frobenius manifold structure on the orbit space $\mathbb{C}^{l+1} / \widetilde{W}^{(k)}\left(C_{l}\right)$. However, unlike the $A$-case, there is an additional freedom in the choice of flat structure on the orbit space, and this freedom is defined in terms of an additional integer $0 \leq m \leq l-k$. Thus, the Frobenius manifold structure on the orbit space-defined by the pair $(k, l)$-depends on the triple $(k, l, m)$. This Frobenius manifold will be denoted $\mathcal{M}_{k, m}\left(C_{l}\right)$.

This construction ${ }^{12}$ also covers the orbit spaces, and their Frobenius manifold structures, for the extended affine Weyl groups $\widetilde{W}^{(k)}\left(B_{l}\right)$ and $\widetilde{W}^{(k)}\left(D_{l}\right)$. The ring of invariant polynomials (freely generated by an appropriate Chevalley-type theorem) for these groups may be obtained from those constructed for the group $\widetilde{W}^{(k)}\left(C_{l}\right)$ by simple changes of variable, and this leads to isomorphic Frobenius manifolds. Thus, it suffices to study the orbit space $\mathbb{C}^{l+1} / \widetilde{W}^{(k)}\left(C_{l}\right)$ with the Frobenius manifold structure $\mathcal{M}_{k, m}\left(C_{l}\right)$.

Furthermore, it was shown in Ref. 12 that $\mathcal{M}_{k, m}\left(C_{l}\right)$ coincides with the Frobenius manifold structure on the space of the cosine-Laurent series of tri-degree $(2 k, 2 m, 2 l)$, namely, functions of the form

$$
\lambda(\tilde{z})=\frac{1}{\left(\cos ^{2} \tilde{z}-1\right)^{m}} \sum_{j=0}^{l} a_{j} \cos ^{2(k+m-j)}(\tilde{z}),
$$

with the choice of primary differential $\omega=d \tilde{z}$.

This space is related, via a Legendre transformation, to a space of $\mathbb{Z}_{2}$-invariant rational functions of the form $\mathbb{Z}_{2}$,

$$
\lambda(z)=z^{2 m}+\alpha_{1} z^{2(m-1)}+\cdots+\alpha_{m}+\sum_{r=1}^{l-(k+m)} \frac{\beta_{r}}{z^{2 r}}+\sum_{s=1}^{k} \frac{\gamma_{s}}{\left(z^{2}-\left(z^{\circ}\right)^{2}\right)^{s}},
$$

with primary differential $\omega=d z$. We denote this space $\mathcal{M}_{m, l-(k+m), k}^{\mathbb{Z}_{2}}$. This space is related, via a Legendre transformation, to the orbit space $\mathcal{M}_{k, l}\left(C_{l}\right)$.

The coefficient $t^{\circ}$, defined by the term

$$
\lambda(z)=\cdots+\frac{z^{\circ} t^{\circ}}{\left(z^{2}-\left(z^{\circ}\right)^{2}\right)}+\cdots
$$

turns out, by evaluating certain residues, to be a flat coordinate, and hence, a change of primary differential is given by

$$
d \tilde{z}=\left\{\partial_{t^{\circ}} \lambda(z)\right\} d z .
$$

Hence (up to an overall constant that may be ignored), $z=i z^{\circ} \cot \tilde{z}$, and this induces the Legendre transformation between the two Frobenius manifolds, i.e., this change in primary differential induces a change of variable that maps (10) to (11). Thus, the Frobenius manifold structures on $\mathcal{M}_{k, l}\left(C_{l}\right)$ and the $\mathbb{Z}_{2}$-graded Hurwitz space $\mathcal{M}_{m, l-(k+m), k}^{\mathbb{Z}_{2}}$ are related by a Legendre transformation.

As in the $A$-case, the twisted Legendre transformation between the corresponding almost-dual manifolds turns out to a normal Legendre transformation. The extended- $\bigvee$-systems may be easily calculated by writing the superpotential in the form

$$
\lambda(z)=\frac{\prod_{i=1}^{l}\left(z^{2}-\left(z^{i}\right)^{2}\right)}{z^{2(l-(k+m))}\left(z^{2}-\left(z^{\circ}\right)^{2}\right)^{2 k}} .
$$

Unlike the $A$-case, the $z_{o}$ variable is not constrained: the flat coordinates for the metric are $z^{i}, i=0, \ldots, l$. This is a special case, with $s=-2(l-(k+m))$ and

$$
\mathbf{k}=\{-2 k, \underbrace{1, \ldots, 1}_{l}\}
$$


of the general superpotential (3).

Lemma 23. The twisted Legendre field $E \circ \frac{\partial}{\partial t^{\circ}}$ is constant in the $\left\{z^{i}\right\}$-variables. Moreover, the field is perpendicular to the space

$$
T V=\operatorname{span}\left\{\frac{\partial}{\partial z^{i}}, i=1, \ldots, l\right\} .
$$

Proof. Since

$$
4 k \frac{\partial \lambda}{\partial t^{\circ}}=\frac{\partial \log \lambda}{\partial z^{o}}
$$

it immediately follows that for all $i=0, \ldots, l$,

$$
g\left(E \circ \frac{\partial}{\partial t^{\circ}}, \frac{\partial}{\partial z^{i}}\right)=g\left(\frac{1}{4 k} \frac{\partial}{\partial z^{\circ}}, \frac{\partial}{\partial z^{i}}\right) .
$$

Hence,

$$
E \circ \frac{\partial}{\partial t^{\circ}}=\frac{1}{4 k} \frac{\partial}{\partial z^{\circ}}
$$

In these variables,

$$
g=\sum_{i=1}^{l}\left(d z^{i}\right)^{2}-2 k\left(d z^{\circ}\right)^{2}
$$

and hence, it follows that the twisted Legendre field is perpendicular to the space TV.

We end by noting two things:

(1) To make the connection with Sec. II,

$$
\begin{aligned}
& T V \text { is spanned by }\left\{\frac{\partial}{\partial w^{i}} \text { for } A \text { type }\right\},\left\{\frac{\partial}{\partial z^{i}} \text { for } B, C, D \text { type }\right\}, \\
& T V^{\perp} \text { is spanned by }\left\{\frac{\partial}{\partial w^{\perp}} \text { for } A \text { type }\right\},\left\{\frac{\partial}{\partial z^{\perp}} \text { for } B, C, D \text { type }\right\} .
\end{aligned}
$$

Hence, the twisted Legendre field $E \circ \frac{\partial}{\partial t^{\circ}}$ is perpendicular to the space $V$ (the constraint in the $A$-case, $\sum_{i=1}^{l+1} w^{i}=0$, is just the manifestation of the standard representation of the $A_{l}$ root system as a hyperplane in $\mathbb{R}^{l+1}$.

(2) Since $\tilde{\partial}$ is constant in these (flat)-coordinates, the twisted Legendre transformation is actually a normal Legendre transformation, and such a normal Legendre transformation has already been performed in Sec. IV.

The choice of original Legendre field $\partial=\partial_{t^{\circ}}$ was very special - others choices would have resulted in a non-constant twisted Legendre field. It may be shown that these special properties come from the fact that $\mu=-\frac{1}{2}$ lies in the spectrum of the underlying Frobenius manifold..$^{18}$

\section{CONCLUSIONS}

As remarked earlier and as is apparent from Fig. 4, an extended $\bigvee$-system, based, say, on the root system $R_{W}$ of a classical Weyl group $W$ of rank $n$, is invariant under the action of $W$. However, on performing a Legendre transformation, one obtains configurations invariant under an extended affine Weyl group of rank $n+1$. It is therefore natural to ask what is the origin of this extra symmetry that does not appear in the extended $\bigvee$-system. The answer lies in the precise nature of the Legendre transformation $\hat{z} \leftrightarrow z$. For example, in the $A_{n}$ example, the perpendicular direction $z_{\perp}$ is invariant under the affine translation,

$$
\hat{z}_{\perp} \mapsto \hat{z}_{\perp}+8 \pi i h_{\beta}(n+1),
$$

since the transformation is exponential. Thus, in the $\hat{z}$-space, one has a symmetry that is not apparent in the original $z$-space. Together with the original action of $W$, one thus obtains an extended affine group action.

The construction in this paper is dependent on the existence of a small orbit, and for exceptional Coxeter groups, such orbits do not exist. However, variants of the construction in this paper do exist: the small orbit condition is sufficient for the construction, but it is certainly not necessary, as the following example shows:

Example 24. Consider the root system of the Coxeter group $F_{4}$,

$$
\mathcal{R}_{F_{4}}=\mathcal{R}_{\text {long }} \cup \mathcal{R}_{\text {short }},
$$


where

$$
\begin{aligned}
\mathcal{R}_{\text {long }} & =\{( \pm 1, \pm 1,0,0), \text { and permutations }\} \\
\mathcal{R}_{\text {short }} & =\{( \pm 1,0,0,0) \text {, and permutations }\} \cup\left\{\left( \pm \frac{1}{2}, \pm \frac{1}{2}, \pm \frac{1}{2}, \pm \frac{1}{2}\right) \text {, and permutations }\right\} .
\end{aligned}
$$

These give the standard almost-dual prepotential

$$
F^{\star}=\frac{1}{4} \sum_{\alpha \in \mathcal{R}_{F_{4}}} \alpha(z)^{2} \log \alpha(z)
$$

with

$$
2 h(z, z)=\sum_{\alpha \in \mathcal{R}_{F_{4}}} \alpha(z)^{2}
$$

and $h=9$, the dual Coxeter number of $F_{4}$. The long roots do not form a small orbit, but it does have the following property: for $w_{1}, w_{2} \in \mathcal{R}_{\text {long }}$ with $w_{1} \neq \pm w_{2}$,

(i) either $w_{1}+w_{2} \in \mathcal{R}_{\text {long }}$ or $w_{1}-w_{2} \in \mathcal{R}_{\text {long }}$

or

(ii) $w_{1}$ and $w_{2}$ are perpendicular.

One can then extend the vectors in $\mathcal{R}_{\text {long }}$ to obtain the extended $\bigvee$-system,

$$
\mathcal{U}=\mathcal{R}_{F_{4}} \cup\left\{ \pm(w+n), w \in \mathcal{R}_{\text {long }}\right\} \cup\{ \pm n\}
$$

with the multiplicities $h_{\alpha}=1, \frac{1}{2}, 3$, respectively. This gives an extended $F_{4}$ solution to the WDVV equations. By construction, the five-dimensional extended configuration is invariant under the Coxeter group $F_{4}$. This configuration was obtained in Ref. 19 by restricting the $E_{8}$ system onto a certain discriminant; in their notation, this is the solution $\left(E_{8}, A_{1}^{3}\right)$.

Whether a similar construction may be applied to the remaining exceptional cases is under investigation. However, the construction in Ref. 11, coupled with almost-duality, guarantees a trigonometric $\bigvee$-system for such exceptional cases (for a specific marked node in Ref. 11, and conjecturally for an arbitrary marked node). Whether such systems are the Legendre-transformed versions of some extended-rational $\bigvee$-system is unknown, though it is natural to conjecture that they are. More generally, a natural question to ask is whether there is some direct map between rational $\bigvee$-systems and trigonometric $\bigvee$-systems, and if not, to find under what conditions it does exist.

Further examples, too, would be of interest. There has been recent work on the classification of $\bigvee$-systems, ${ }^{20,21}$ and it would be interesting to see if the extended version of these systems exists. One could ask, for example, how the matroid for the extended systems can be constructed from the matroid of the original system. The complex-reflection/Shephard group examples recently constructed in Ref. 20 would be a good place to start: these already have interesting symmetry groups automatically built into their construction.

The small orbit property also provides an explanation of the ad hoc construction of elliptic $\bigvee$-systems ${ }^{22}$ and elliptic solutions to the WDVV equations. These solutions have, as their leading term, a function that by itself is a solution to the WDVV equations of the form (1), but an irregular orbit had to be added, which was unclear. It turns out that the irregular orbits are precisely small orbits. One observation coming from these results is that for Coxeter groups, the existence of an irreducible quartic invariant polynomial is equivalent to the existence of a small orbit. Whether this is significant or just accidental is unclear.

The WDVV equations and the rational solutions come from the commutativity, or zero-curvature relations, for the deformed connection,

$$
\nabla_{a}=\partial_{a}+\kappa \sum_{\alpha \in \mathcal{U}} \frac{(\alpha, a)}{(\alpha, z)} \alpha^{\vee} \otimes \alpha
$$

The construction in this paper can also be thought of in terms of extending such a connection into a dimension higher. The geometry of such a construction also deserves to be studied. Such questions also appear in the Hurwitz space description. For example, consider the $A_{n}$-example and its extension. This construction corresponds to adding an extra term to the superpotential,

$$
\left.\left.\prod_{i=0}^{n}\left(z-z^{i}\right)\right|_{\sum_{i=1}^{n} z^{i}=0} \mapsto \frac{\prod_{i=0}^{n}\left(z-z^{i}\right)}{\left(z-z^{\circ}\right)^{k}}\right|_{\sum_{i=0}^{n} z^{i}-n z^{\circ}=0},
$$

and the geometry of Hurwitz theory requires that $k \in \pm \mathbb{N}$. Algebraically, this restriction is not required in the $\bigvee$-system. It is here that the sign of $k$ affects the geometry (but not the algebra). If $k$ is positive, this generates the extended affine Weyl orbit space and a solution that is almost dual to the corresponding Frobenius manifold. If $k$ is negative, the superpotential no longer has a pole but a multiple root. This corresponds 
to the induced Frobenius structures on discriminant surfaces with a larger manifold. ${ }^{23}$ That such induced structures on discriminant generate solutions to the WDVV equations of the form (1) was proved in Ref. 19.

The flat connection is, as many authors have pointed out, very similar in form to the celebrated Dunkl operator. It would be of interest to see if these ideas-particularly, the idea of a Legendre transform-could be applied to such a Dunkl operator. Finally, root systems and $\checkmark$-systems appear in many other places in mathematical physics: in the theory of Calogero-Moser and Schrödinger operators, for example, Ref. 16, and there are rational and trigonometric versions of both of these. Whether these are connected by a suitable Legendre transformation is unknown.

All these questions require further work.

\section{ACKNOWLEDGMENTS}

Richard Stedman was funded by the ESPRC Doctoral Training Grant No. EP/K503058/1.

\section{DATA AVAILABILITY}

Data sharing is not applicable to this article as no new data were created or analyzed in this study.

\section{REFERENCES}

${ }^{1}$ A. Marshakov, A. Mironov, and A. Morozov, “More evidence for the WDVV equations in N = 2 SUSY Yang-Mills theories,” Int. J. Mod. Phys. A 15, 1157-1206 (2000).

${ }^{2}$ B. A. Dubrovin, "On almost duality for Frobenius manifolds," in Geometry, Topology and Mathematical Physics, American Mathematical Society Translations: Series 2 Vol. 212 (American Mathematical Society, Providence, RI, 2004), pp. 75-132.

${ }^{3}$ T. Bridgeland, "Spaces of stability conditions," in Algebraic Geometry-Seattle 2005, Part 1, Proceedings of Symposia in Pure Mathematics Vol. 80 (American Mathematical Society, Providence, RI, 2009), pp. 1-21.

${ }^{4}$ A. Bellucci, A. V. Galajinsky, and E. Latini, "New insight into WDVV equation," Phys. Rev. D 71, 044023 (2005).

${ }^{\mathbf{5}}$ O. Lechtenfeld, K. Schwerdtfeger, and J. Thürigen, “ $N=4$ multi-particle mechanics, WDVV equation and roots," SIGMA 7, 023 (2011).

${ }^{6}$ A. P. Veselov, "Deformations of the root systems and new solutions to generalised WDVV equations," Phys. Lett. A 261, 297-302 (1999).

${ }^{7}$ V. Serganova, "On generalizations of root systems," Commun. Algebra 24, 4281-4299 (1996).

${ }^{8}$ B. A. Dubrovin, "Geometry of 2D topological field theories," in Integrable Systems and Quantum Groups (Montecatini, Terme, 1993), Lecture Notes in Mathematics Vol. 1620 (Springer, 1993), pp. 120-348.

${ }^{9}$ M. Feigin, “Trigonometric solutions of WDVV equations and generalised Calogero-moser-Sutherland systems," SIGMA 5, 088 (2009).

${ }^{10}$ M. Alkadhem and M. Feigin, “Trigonometric $\bigvee$-systems and solutions of WDVV equations,” J. Phys. A: Math. Theor. 54, 024002 (2020).

${ }^{11}$ B. A. Dubrovin and Y. J. Zhang, "Extended affine Weyl groups and Frobenius manifolds," Compos. Math. 111(2), 167-219 (1998).

${ }^{12}$ B. A. Dubrovin, I. A. B. Strachan, Y. Zhang, and D. Zuo, "Extended affine Weyl groups of BCD type, Frobenius manifolds and their Landau-Ginzburg superpotentials," Adv. Math. 351, 897-946 (2019).

${ }^{13}$ A. Riley and I. A. B. Strachan, “A note on the relationship between rational and trigonometric solutions of the WDVV equations,” J. Nonlinear Math. Phys. 14(1), 82-94 (2007).

${ }^{14}$ R. Stedman, "Deformations, extensions and symmetries of solutions to the WDVV equations," Ph.D. thesis, University of Glasgow, 2016.

${ }^{15}$ M. A. Feigin and A. P. Veselov, On the Geometry of $\bigvee$-systems, American Mathematical Society Translations: Series 2 Vol. 214 (American Mathematical Society, Providence, RI, 2008), pp. 111-123.

${ }^{16}$ A. N. Sergeev and A. P. Veselov, “Deformed quantum Calogero-Moser systems and Lie super-algebras,” Commun. Math. Phys. 245, 249-278 (2004).

${ }^{17}$ K. Saito, "On a linear structure of the quotient variety by a finite reflection group," Publ. Res. Inst. Math. Sci. 29, 535 (1979).

${ }^{18}$ R. Stedman and I. A. B. Strachan, "Generalised Legendre transformation and symmetries of the WDVV equations," J. Phys. A: Math. Theor. 50, 095202 (2017).

${ }^{19}$ M. A. Feigin and A. P. Veselov, "Logarithmic Frobenius structures and Coxeter discriminants," Adv. Math. 212(1), 143-162 (2004).

${ }^{20}$ A. Arsie and P. Lorenzoni, “Complex reflection groups, logarithmic connections and bi-flat F-manifolds," Lett. Math. Phys. 107, 1919 (2017).

${ }^{21}$ V. Schreiber and A. P. Veselov, "On deformation and classification of V-systems," J. Nonlinear Math. Phys. 21(4), 543-583 (2014).

${ }^{22}$ I. A. B. Strachan, "Weyl groups and elliptic solutions of the WDVV equations," Adv. Math. 224(1), 1801-1838 (2010).

${ }^{23}$ I. A. B. Strachan, "Frobenius manifolds: Natural submanifolds and induced bi-Hamiltonian structures," Differ. Geom. Appl. 20(1), 67-99 (2004). 